Revista Colombiana de Obstetricia y Ginecología Vol. 61 No. 1 • $2010 \bullet(12-17)$

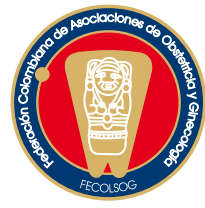

INVESTIGACIÓN ORIGINAL

\title{
VIGILANCIA EPIDEMIOLÓGICA DE CICLOPÍA EN EL HOSPITAL UNIVERSITARIO DEL VALLE, CALI (COLOMBIA) 2004-2008
}

\section{Epidemiological surveillance of cyclopia in the Hospital Universitario del Valle, Calli, Colombia 2004-2008}

Wilmar Saldarriaga, M.D., MSc.*

Recibido: septiembre 17/09 - Aceptado: febrero 1/10

\section{RESUMEN}

Objetivos: determinar y analizar la prevalencia de ciclopía en el Hospital Universitario del Valle (HUV) y buscar en los casos los factores etiológicos descritos en la literatura.

Metodología: el presente es un estudio de corte transversal analítico, ensamblado a partir del sistema de vigilancia epidemiológica de malformaciones congénitas en el HUV y bajo la metodología del Estudio Colaborativo Latinoamericano de Malformaciones Congénitas (ECLAMC), se detectaron los casos de ciclopía acontecidos entre diciembre de 2004 y abril de 2008, se obtuvo el número de nacimientos y se encontró la prevalencia de ciclopía en el HUV. Gracias a un formato de entrevista del ECLAMC, se buscaron en las pacientes los factores que según la literatura se asocian con la ocurrencia de ciclopía. Se tomó fotos de cada uno de los casos.

Resultados: durante el período comprendido entre diciembre de 2004 y abril de 2008 se presentaron 18.224 nacimientos en el HUV. De éstos, 8 presentaron ciclopía con una prevalencia de

* Médico Gineco-obstetra. Ciencias Básicas Médicas, Énfasis en Embriología y Genética, (MACOS) Grupo de Malformaciones Congénitas Perinatales y Dismorfología. Profesor en los Departamentos de Morfología, Ginecología y Obstetricia, Facultad de Ciencias de la Salud, Universidad del Valle. Cali. (Colombia). Ginecólogo en el Hospital Universitario del Valle y en la Fundación Valle del Lili. Cali (Colombia).

Correo electrónico:wsaldarriaga0608@yahoo.com
1 en 2.278 nacimientos (43,8 por 100.000). Todos los casos se clasificaron según Meyer como tipo I y 3 casos presentaron etmocefalea. Se encontró exposición a misoprostol, alcohol y marihuana en un caso; diabetes en otro, marihuana en otro; un caso presentó trisomia 13 y otro en que no se obtuvo cariotipo los hallazgos sugirieron trisomía 13.

Conclusiones: La ciclopía en Cali y el Valle del Cauca es considerada un evento endémico. Por lo cual, se necesita un sistema de vigilancia epidemiológico de malformaciones congénitas, holoprosencefalia y ciclopía para Cali y el Valle del Cauca. Además, se necesitan estudios controlados sobre factores de riesgo individuales y colectivos.

Palabras clave: ciclopía, holoprosencefalia, endemia, ECLAMC.

\section{SUMMARY}

Objectives: identifying and analysing cyclopia prevalence in the Hospital Universitario del Valle and searching for the aetiological factors described in the literature in the cases so found.

Methodology: this was a prospective study of cases and controls regarding cases of cyclopia occurring between December 2004 and April 2008 which were detected via HUV's congenital malformation epidemiological surveillance system, using Latin-American Collaborative Study of Congenital Malformations (ECLAMC) methodology. The number 
of births and cyclopy prevalence in the HUV were obtained. ECLAMC's interview form was used for searching for factors associated by the literature with cyclopy occurring. Photos were obtained in all cases. Results: there were 18,224 births in the HUV from between December 2004 and April 2008; 8 of these presented cyclopy, giving a 1 in 2,278 births prevalence (43.8 per 100,000). All cases were classified as being Meyer type I. Three cases suffered from etmocephaly. Exposure to misoprostol, alcohol and marijuana was found in one case; diabetes in another; and marijuana in a third one. A case of trisomy 13 was presented and another case in which findings suggested trisomy 13 , the karyotype was not obtained.

Conclusions: cyclopy in Cali and Valle del Cauca are endemic events. A congenital malformation, holoprosencephaly and cyclopy epidemiological surveillance system is needed for Cali and Valle del Cauca area.

Key words: cyclopia, holoprosencephaly, endemic, ECLAMC.

\section{INTRODUCCIÓN}

La ciclopía es una malformación congénita poco frecuente, es el grado máximo del espectro de presentación fenotípica de la holoprosencefalia (HPE), producto de una secuencia que se inicia con un defecto en la división del prosencéfalo que produce, en grado variable, la pobre formación de los hemisferios y las estructuras de la línea media del cerebro. De este modo, la HPE puede ser lobar, semilobar o alobar. ${ }^{1,2}$

Por su parte, la ciclopía se presenta en los casos de HPE alobar y no hay formación de los hemisferios cerebrales dejando como resultado una única estructura cerebral, el prosencéfalo, con un ventrículo único y los tálamos fusionados. Además, se produce una alteración de los derivados embriológicos de la eminencia fronto-nasal y un desarrollo defectuoso de la línea media de la cara, con fusión de las órbitas de los globos oculares pudiendo producir un único ojo o dos pero fusionados dentro de una única cámara orbital. ${ }^{1-3}$ Existen otros defectos faciales menos severos relacionados con la holoprosencefalia que pueden afectar la línea media de la cara, sin producir ciclopía, pero sí un hipotelorismo con una severidad variable, todos estos incluidos en la clasificación DeMyer. ${ }^{1-5} \mathrm{La}$ prevalencia a nivel mundial de ciclopía es de 1 a 3 en 100.000 nacimientos, siendo mayor en mujeres que en hombres 3:1., $3-8$

La etiología de esta malformación es heterogénea y solamente se encuentra la causa entre el 15\% y el 20\% de los casos. Las causas identificadas son: monogénicas, cromosómicas y teratogénicas. Entre los patrones de herencia monogénicos no sindrómicos se encuentran más comúnmente los dominantes con penetrancia incompleta y expresividad variable y menos común los de transmisión recesiva y ligados al cromosoma X. ${ }^{1,2,8}$

Asimismo, se han encontrado mutaciones en 9 genes como causantes de HPE: SHH, PTCH, GLI2, DHCR7, ZIC2, TDGF1, TGIF, FAST1 y SIX3. Las funciones defectuosas de las proteínas codificadas por estos genes están relacionadas en las vías de señalización del Sonic Hedgehog., ${ }^{9}$

Igualmente, la diabetes materna es el factor teratogénico más importante. El consumo de etanol, ácido retinoico y salicilatos durante el primer trimestre del embarazo también han sido reportados, ${ }^{1,8,11-14}$ y la alteración cromosómica numérica más frecuentemente relacionada es la trisomía 13. ${ }^{1,14}$ Sin embargo, en los defectos congénitos de etiología heterogénea como la HPE, la importancia de la interacción entre factores genéticos y ambientales aún no ha sido resuelta.

Por esta razón, es importante que el especialista en gineco-obstetricia y en medicina materno-fetal y el personal médico en general, tengan en cuenta que los defectos congénitos de muy baja prevalencia como la HPE y la ciclopía deben ser sospechados en las ecografías básicas y precisados en las de alta complejidad. Además, una vez que éste se enfrente con un diagnóstico prenatal y/o postnatal 
de holoprosencefalia y ciclopía, es necesario llevar un protocolo de manejo etiológico para así poder ofrecer respuestas a los interrogantes de la pareja y una consejería reproductiva adecuada. Específicamente, en el Valle del Cauca se han hecho reportes de cluster de ciclopía, por lo cual se hace indispensable realizar estudios de prevalencia duraderos en el tiempo y analizar sus resultados.

Por lo tanto, este estudio tiene como objetivo determinar y analizar la prevalencia de ciclopía en el Hospital Universitario del Valle (HUV) y buscar en los casos los factores etiológicos descritos en la literatura.

\section{METODOLOGÍA}

Diseño: el presente es un estudio de corte transversal analítico, ensamblado a partir del sistema de vigilancia epidemiológica de malformaciones congénitas en el HUV y bajo la metodología del Estudio Colaborativo Latinoamericano de Malformaciones Congénitas (ECLAMC), el cual es un estudio prospectivo de casos y controles.

Población: se incluyeron los recién nacidos en el Hospital Universitario del Valle (HUV), vivos o muertos y con más de $500 \mathrm{~g}$, entre diciembre de 2004 y abril de 2008. A partir de este punto, se determinaron y analizaron los casos que presentaron ciclopía, los cuales fueron definidos como recién nacidos, vivos o muertos, con más de 500 g, con única cavidad orbitaria y con uno o dos globos oculares fusionados.

El HUV es un hospital de nivel III y IV de referencia en obstetricia, de la red pública del suroccidente colombiano. Atiende entre $6.000 \mathrm{y}$ 7.000 nacimientos por año de mujeres de estrato socioeconómico 1, 2 y en menor proporción del estrato 3, del régimen subsidiado, con patologías de alta complejidad materno-fetal, siendo esta una población seleccionada.

Procedimiento: a partir de la base de datos del sistema de vigilancia epidemiológica de malformaciones congénitas en el HUV se seleccionaron las fichas que presentaban como diagnóstico ciclopía y se determinaron las fechas de nacimiento correspondientes. Enseguida, se procedió a revisar si al feto se le había hecho cariotipo, autopsia fetal y la descripción de los hallazgos dismórficos y se mostraron las fotos tomadas. Con base en el informe mensual de nacimientos entre diciembre de 2004 y abril de 2008 se construyó el denominador de la prevalencia.

Variables a medir: se evaluaron la edad materna, el municipio de residencia en el primer trimestre del embarazo, la exposición a la diabetes, al alcohol, a la marihuana, a los psicofármacos, a los medicamentos en general, al misoprostol, a los salicilatos y los antecedentes familiares de malformaciones congénitas y la HPE.

Análisis estadístico: se describieron tanto la prevalencia en el HUV como la frecuencia absoluta de los factores de riesgo.

Consideraciones éticas: en todos los casos los padres aceptaron, por medio de la firma de un consentimiento informado, la inclusión del feto en la metodología del ECLAMC, la cual incluye la recolección de datos y la toma de fotografías. De igual modo, en aquellos casos en que se realizó autopsia fetal, éstas fueron autorizadas por los padres con la firma del consentimiento especial según los protocolos del HUV para dicho procedimiento.

\section{RESULTADOS}

Durante el período comprendido entre diciembre de 2004 y abril de 2008 se presentaron 18.224 nacimientos. De éstos, 8 presentaron ciclopía con una prevalencia de 1 en 2.278 nacimientos (43,8 por 100.000). Todos los casos se clasificaron según Meyer en tipo I.

En la búsqueda de factores relacionados con las madres de los recién nacidos con ciclopía en el HUV, se encontró que el primer caso presentaba diabetes, y el tercero mostraba antecedentes de consumo de alcohol, misoprostol y marihuana durante el primer trimestre del embarazo. En la madre del cuarto caso se encontró consumo de 
Figura 1. Los 8 casos de ciclopía. En la parte superior de cada figura se observa la fecha de nacimiento, día, mes y año, pudiéndose correlacionar con la columnas de la tabla 1. Los casos 2, 5 y 8 presentan probóscide, configurando una etmocefalia y los casos 5 y 8 presentan dos ojos fusionados clasificados como cíclopes con sinotia.
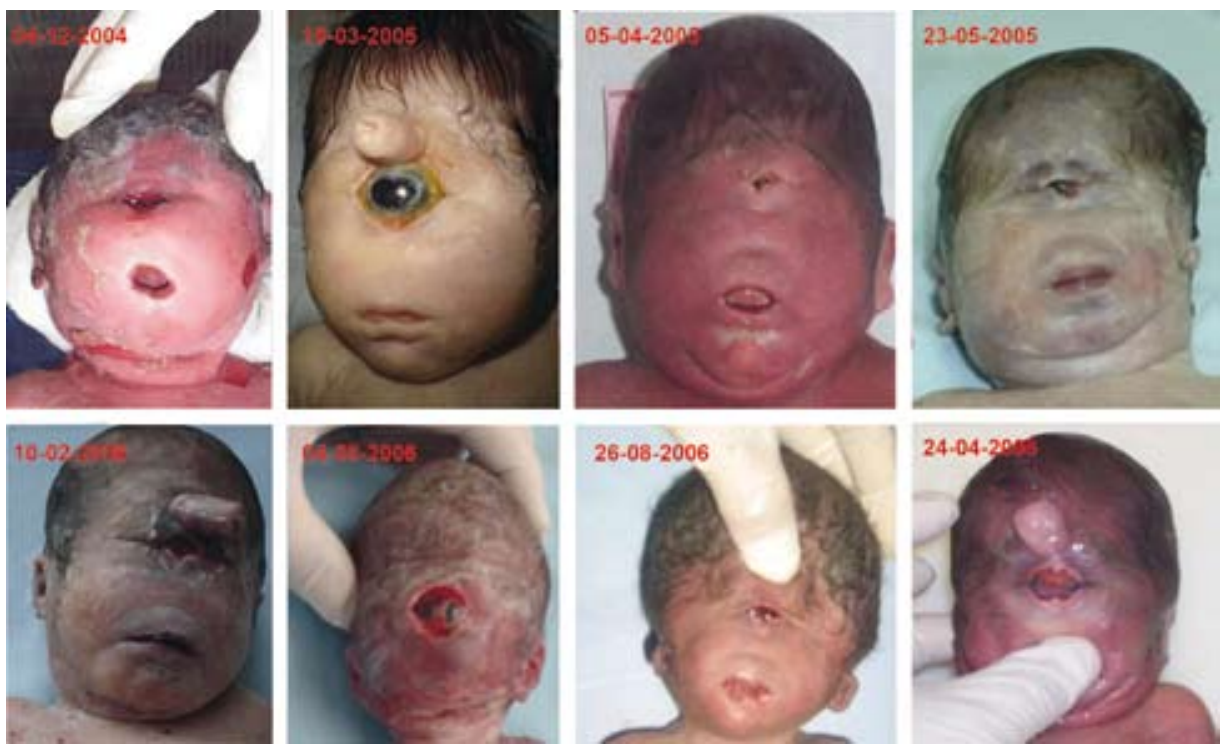

marihuana también para el primer trimestre del

Además, se realizó cariotipo en 6 de los 8 casos; embarazo. Los aspectos demográficos y los factores asociados que fueron estudiados se describen en la tabla 1 . de éstos, 1 presentó $47 \mathrm{XX}+13$. En uno de los casos en que no se realizó cariotipo se encontró polidactilia postaxial en las cuatro extremidades,

\begin{tabular}{|c|c|c|c|c|c|c|c|c|}
\hline $\begin{array}{l}\text { Fecha de } \\
\text { nacimiento }\end{array}$ & 04-12-2004 & $10-03-2005$ & 05-04-2005 & 23-05-2005 & $10-02-2006$ & 04-08-2006 & 26-08-2006 & 24-04-2007 \\
\hline Edad materna & 35 & 19 & 19 & 24 & 30 & 23 & 22 & 24 \\
\hline $\begin{array}{l}\text { DeMyer tipos } \\
\text { I a IV }\end{array}$ & Único ojo & $\begin{array}{l}\text { Único ojo. } \\
\text { Prosbocis } \\
\text { etmocefalia. }\end{array}$ & Único ojo & Único ojo & $\begin{array}{c}2 \text { ojos } \\
\text { fusionados. } \\
\text { Sinotia. }\end{array}$ & $\begin{array}{l}\text { Único ojo. } \\
\text { Proboscis } \\
\text { etmocefalia. }\end{array}$ & Único Ojo & $\begin{array}{l}2 \text { ojos fu- } \\
\text { sionados. } \\
\text { Sinotia, } \\
\text { proboscis } \\
\text { etmoce- } \\
\text { falia. }\end{array}$ \\
\hline Otras anomalías & - & Mielosquisis & Microtia & - & - & - & Microtia & $\begin{array}{l}\text { Polidac- } \\
\text { tilia }\end{array}$ \\
\hline $\begin{array}{l}\text { Municipio de } \\
\text { residencia } 1 \text { er } \\
\text { trimestre }\end{array}$ & Guacarí & Toro & Cali & Cali & Cali & Yumbo & Cali & Cali \\
\hline Cariotipo & - & $46 \mathrm{XX}$ & $46 \mathrm{XX}$ & $\begin{array}{c}46 \text { XX } 16 \\
\text { h+ }\end{array}$ & $47 X X+13$ & $46 \mathrm{XX}$ & $46 \mathrm{XX}$ & - \\
\hline Autopsia & + & + & + & + & + & + & + & - \\
\hline $\begin{array}{l}\text { Factores } \\
\text { de riesgo }\end{array}$ & Diabetes II & - & $\begin{array}{c}\text { Miso- } \\
\text { prostol, } \\
\text { marihuana, } \\
\text { alcohol. }\end{array}$ & Marihuana & - & $\begin{array}{l}2 \text { abortos } \\
\text { previos. }\end{array}$ & - & - \\
\hline
\end{tabular}


lo cual sugiere que la causa probablemente sería una trisomía 13.

De otra parte, ninguna de las pacientes mostraba antecedentes familiares con características fenotípicas relacionadas con la HPE para sospechar un origen hereditario. Sin embargo, no se realizaron estudios de biología molecular para encontrar mutaciones en los genes específicos ligados a la holoprosencefalia y ciclopía.

\section{DISCUSIÓN}

La prevalencia de ciclopía en el Hospital Universitario del Valle en Cali (Colombia) fue de 43,8 en 100.000 nacimientos. Aproximadamente entre 14 y 43 veces más que lo reportado en la literatura.

Los primeros 4 casos nacieron en un período de 170 días y fueron interpretados como un cluster de ciclopía en el Valle del Cauca. Además, durante ese mismo lapso de tiempo, pero en 54 días, nacieron 4 casos de sirenomelia que también fueron interpretados como un cluster para la ciudad de Cali. ${ }^{15-18}$

Ahora bien, tomando en cuenta las características del HUV, es probable que se encuentren recién nacidos con defectos congénitos de muy baja prevalencia (ciclopía) como consecuencia del efecto de la referencia, por lo cual no sería comparable con los datos presentes en la literatura, los cuales toman los nacimientos de maternidades de alta y baja complejidad como los del ECLAMC. ${ }^{7}$

Sin embargo, de los 8 casos, 5 provenían de Cali y 3 de otros municipios del Valle del Cauca. En el período de tiempo estudiado hubo 70.243 nacimientos en Cali y 193.780 en el Valle del Cauca, mostrando prevalencias para ciclopía de 7,12 y 4,12 por 100.000 nacimientos, respectivamente. Prevalencias que siguen siendo elevadas al compararlas con la literatura. Por consiguiente, estos hallazgos sugieren que la ciclopía en dichos lugares podría ser un evento endémico.

Por otra parte, al ser la ciclopía el grado máximo del espectro de presentación clínica de la holoprosencefalia y que un porcentaje importante de estos fetos mueren, presentándose como abortos espontáneos, no alcanzarían una edad gestacional suficiente para ser diagnosticados por medio de una ecografía o al ser examinados tras el aborto. Asimismo, en casos menos severos que son compatibles con la vida, tanto el recién nacido como los niños o adultos pueden ser difíciles de diagnosticar porque los mayores defectos serían intracerebrales. Por tanto, los datos de prevalencias elevadas de ciclopía sugerirían indirectamente que la prevalencia de la holoprosencefalia en general podría ser mucho mayor que la esperada, siendo la ciclopía solamente el hallazgo identificable.

El análisis descriptivo sugiere que ante el diagnóstico de la HPE y/o ciclopía se debe realizar una curva de tolerancia a la glucosa, cariotipo y pruebas de biología molecular en alguno de los productos de la gestación (vellosidad corial, líquido amniótico, sangre fetal o del recién nacido), en búsqueda de diabetes materna, alteraciones cromosómicas y mutaciones en los genes relacionados, además de un heredograma y una entrevista minuciosa preguntando por exposición al alcohol, salicilatos, misoprostol y otros fármacos específicamente 3 meses antes y después de la probable concepción del embrión o feto con HPE. En cuanto a la marihuana, este es un factor que no se ha relacionado con casos de HPE pero sí en otras malformaciones como gastrosquisis y por haberse encontrado en 2 de los 8 casos de ciclopía aquí reportados se sugiere también sea indagado específicamente.

\section{CONCLUSIÓN}

Los hallazgos aquí reportados sugieren que la ciclopía en Cali y el Valle del Cauca son un evento endémico. Por ende, se necesita reforzar el sistema de vigilancia epidemiológico de malformaciones congénitas, para la detección de los casos de holoprosencefalia y ciclopía para Cali y el Valle del Cauca. Además, se necesitan estudios controlados sobre factores de riesgos individuales y colectivos, ambientales y genéticos, que pudieran precipitar la aparición de casos de HPE y ciclopía. 


\section{AGRADECIMIENTOS}

Al grupo de vigilancia epidemiológica de malformaciones congénitas en el Hospital Universitario del Valle, al grupo de investigación MACOS, a todos los residentes de ginecología y obstetricia y especialmente a la Dra. Carolina Isaza.

\section{REFERENCIAS}

1. Shiota K, Yamada S, Komada M, Ishibashi M. Embryogenesis of holoprosencephaly. Am J Med Genet A 2007;143A:3079-87.

2. Yamada S. Embryonic holoprosencephaly: pathology and phenotypic variability. Congenit Anom (Kyoto) 2006;46:164-71.

3. Bianchi D, Crombleholme T, D Alton M. Fetology. New York: McGraw Hill; 2000 p. 493-98.

4. DeMyer WE. Holoprosencephaly (cyclopiaarhinencephaly). En: Vinken PJ, Bruyn GW, editors. Handbook of clinical neurology. Amsterdam: North Holland Publishing Company. 1977. p. 431-78.

5. DeMyer W, Zeman W, Palmer CG. The face predicts the brain: diagnostic significance of median facial anomalies for holoprosencephaly (arhinencephaly). Pediatrics 1964;34:256-63.

6. Källén B, Castilla EE, Lancaster PA, Mutchinick O, Knudsen LB, Martínez-Frías ML, et al. The cyclops and the mermaid: an epidemiological study of two types of rare malformation. J Med Genet 1992;29:30-5.

7. Castilla EE, Orioli IM. ECLAMC: The Latin American collaborative study of congenital malformations. Community Genet 2004;7:76-94.

8. Kniffin C, McKusick V. Holoprosencephaly, OMIM ${ }^{\circledR}$. Johns Hopkins University, Baltimore. [en línea] 1986 Mar 06 [Visitado en 2009 Sept 12]. Disponible en: http://www.ncbi.nlm.nih.gov/entrez/dispomim. cgi? id $=236100$

9. Cohen MM Jr. The hedgehog signaling network. Am J Med Genet A 2003;123A:5-28.

10. Dubourg C, Bendavid C, Pasquier L, Henry C, Odent S, David V. Holoprosencephaly. Orphanet J Rare Dis 2007;2:8.

11. Münke M. Clinical, cytogenetic, and molecular approaches to the heterogeneity of holoprosencephaly. Am J Med Genet 1989;34:237-45.

12. Martínez-Frías ML, Bermejo E, Rodríguez-Pinilla E, Prieto L, Frías JL. Epidemiological analysis of outcomes of pregnancy in gestational diabetic mothers. Am J Med Genet 1998;78:140-5.

13. Cohen MM Jr, Shiota K. Teratogenesis of holoprosencephaly. Am J Med Genet 2002;109:1-15.

14. Pachajoa H, Tabares K, Quintero JC, Saldarriaga W, Isaza C. Diagnóstico prenatal de ciclopía asociado con trisomía 13. Colomb Med 2008;39:80-5.

15. Solano AF, Saldarriaga W, Isaza C, Mastroiacovo P, Castilla EE. Foco epidémico de sirenomelia en Cali, Colombia. Informe de 4 casos en el Hospital Universitario del Valle en 54 días. Colomb Med 2006;37:213-8.

16. Saldarriaga W, Isaza C, Mastroiacovo P, Castilla EE. Ciclopía en el Hospital Universitario del Valle: reporte de cuatro casos nacidos y revisión de la literatura. Rev Colomb Obstet Ginecol 2007;58:70-7.

17. Orioli IM, Mastroiacovo P, López-Camelo JS, Saldarriaga W, Isaza C, Aiello H, et al. Clusters of sirenomelia in South America. Birth Defects Res A 2009;85:112-8.

18. Castilla EE, Mastroiacovo P, López-Camelo JS, Saldarriaga W, Isaza C, Orioli IM. Sirenomelia and cyclopia cluster in Cali, Colombia. Am J Med Genet A 2008;146A:2626-36. 